\title{
A clinical evaluation of anorectal pressure studies in the diagnosis of Hirschsprung's disease
}

\author{
IAN AARONSON AND H. H. NIXON \\ From the Hospital for Sick Children, Great Ormond Street, London
}

SUMMARY Stimulation of the rectum by stretch results, in the normally innervated bowel, in the reflex relaxation of the internal anal sphincter. The absence of this rectosphincteric reflex in Hirschsprung's disease forms the basis of a simple and safe diagnostic test. Our experience over two years using a simplified air-filled pressure probe system is described, based on the examination of 267 infants and children with abnormal bowel function, of whom 114 were suffering from Hirschsprung's disease.

A review of the last 100 consecutive patients examined shows an overall diagnostic reliability of $85 \%$. This was of the same order as that of a barium enema in these patients, and indeed of obtaining the correct diagnosis on the first rectal biopsy. It has proved easier to exclude the disease $(90 \cdot 8 \%)$ than to confirm its presence $(74.3 \%)$, and has been found to be more reliable than the barium enema in the diagnosis of ultrashort segment disease. The commoner pitfalls in the interpretation of the trace are described.

We consider that this investigation justifies a place in the routine investigation of Hirschsprung's disease alongside the barium enema and rectal biopsy, and is of particular value as a simple screening test in outpatients.

The ever increasing awareness of the importance of the early recognition of Hirschsprung's disease (Nixon, 1964) has focused attention on improved methods of diagnosis. This is usually confirmed by rectal biopsy (Swenson, Fisher, and MacMahon, 1955; Bodian, 1960) or barium enema examination (Fraser and Wilkinson, 1967). However, the wide variety of clinical presentations of Hirschsprung's disease, and the uncertainty and hazards (Swenson et al, 1955; Lillie, 1969) of the present diagnostic methods makes a simple screening test desirable.

The observation in normal patients that the internal anal sphincter relaxes with a rise in rectal tension (Gowers, 1878) and that in Hirschsprung's disease this rectosphincteric reflex is absent (Callaghan and Nixon, 1964), and, furthermore that the aganglionic segment of bowel exhibits abnormal motility (Swenson, Rheinlander, and Diamond, 1949; Hiatt, 1951) has led to the proposal of the use of anorectal pressure measurement as a diagnostic test (Lawson and Nixon, 1967; Schnaufer, Talbert, Haller, Reid, Tobon, and Schuster, 1967).

A simplification of the original equipment developed by Lawson and Nixon (1967) for continuous Received for publication 24 November 1971. recording of the anorectal pressure profile has been in routine clinical use for over two years, and we now report our experience and the diagnostic reliability we have achieved.

\section{Clinical Material}

In the course of the development of our technique we have accumulated experience from 267 patients referred with abnormal bowel function, of whom 114 were suffering from Hirschsprung's disease. In addition, 30 patients who were quite asymptomatic were also studied. As our skill in both the method of examination and the interpretation of our findings has inevitably improved with experience, we have confined our review to the last 100 consecutive patients referred.

The ages of the patients ranged from 3 days to 9 years. Seventeen patients were examined within the first two weeks of life, and a further 38 were under 1 year old. Two patients were premature and a further two suffered from Down's syndrome.

The commonest causes for referral were chronic constipation or intestinal obstruction. Six patients were examined after episodes of diarrhoea associated 
with abdominal distension, and two infants after spontaneous perforation of the bowel.

In all cases, the final diagnosis was unknown at the time of our examination. Thirty-five patients were subsequently proven to have Hirschsprung's disease, and 65 a variety of conditions, which included acquired megacolon, anal stenosis, functional intestinal obstruction, meconium ileus, and bowel atresia.

\section{Method}

The air-filled balloon system devised by Lawson and Nixon (1967) has been simplified to record only the data essential to diagnosis. The probe, made in our own laboratory, is $0.3 \mathrm{~cm}$ in diameter and contains two rubber-covered chambers each $1 \mathrm{~cm}$ long, with a latex balloon mounted $5 \mathrm{~cm}$ from the upper chamber on a flexible connector. Air lines are connected via three pressure transducers to an ultraviolet light paper recorder (S.E. U.V. Recorder 3006, S.E. Laboratories Engineering Ltd). Simple surface electrodes are applied to the skin with adhesive tape as close to the anal verge as possible, and record voluntary muscle activity.

Calibration is performed in an air cylinder, the pressure being increased and then decreased in stepwise increments by means of a hand-held bulb with a release valve. With the patient in the left lateral position, the probe is inserted into the rectum and steadied so that the lower chamber lies just within the anal canal. Thus the upper chamber records from the internal sphincter and the lower predominantly from the external sphincter (Fig. 1). When resting conditions obtain, usually after four or five minutes, the rectal balloon is distended by increments of air introduced from a syringe by an assistant. Pressure patterns from different levels of the rectum can be elicited by moving the position of the probe. The bowel must be examined in the unprepared state.

Infants require to be comforted with a dummy or feeding bottle, but older children are quite undisturbed. Sedation has, therefore, been found unnecessary. Only on three occasions did the patient's apprehension prevent a satisfactory record from being obtained.

The apparatus is mounted on a compact trolley and can readily be taken to the cot side (Fig. 2).

\section{Characteristic Anorectal Pressure Profiles}

We record pressure patterns in the rectum, internal sphincter, and external sphincter zones during three phases of observations, namely, at rest, on inflation of the rectal balloon and on deflation.
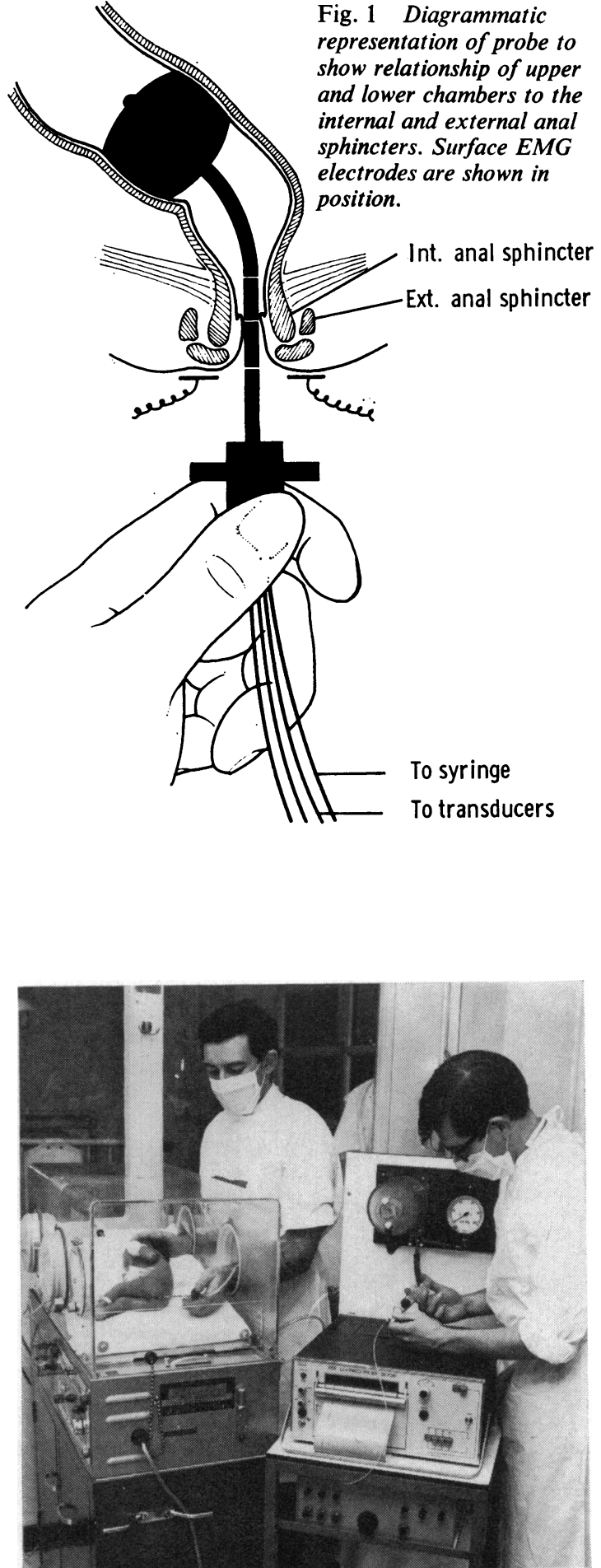

Fig. 2 Anorectal testing in progress on a neonate with intestinal obstruction. 
NORMAL PATIENTS

In the resting trace, the rectum may show occasional very low amplitude contraction waves (Fig. 3). The internal sphincter zone resting pressure may lie between 32 and $58 \mathrm{~cm}$ water with a mean of $38 \mathrm{~cm}$ water and has a quite characteristic rhythmical activity of 10 to $13 / \mathrm{min}$ with an amplitude of 8 to 11 $\mathrm{cm}$ water. The external sphincter zone shows spikes of irregular activity.

On distending the balloon using $10 \mathrm{ml}$ of air in infants and $25 \mathrm{ml}$ in older children, the rectum immediately responds with a transient rise in pressure lasting 15-20 seconds. At the same time the internal sphincter rhythmical activity is depressed or abolished, and its pressure falls by 15 to $20 \mathrm{~cm}$, the duration of this relaxation coinciding with the rectal wave. The external sphincter shows a transient increased activity. At a critical volume (about $25 \mathrm{ml}$ in infants and $150 \mathrm{ml}$ in older children), the internal sphincter remains depressed, the external sphincter relaxes and expulsive efforts are made, producing rapid fluctuation in pressure.

On deflation, the internal sphincter shows a return of rhythmical activity which may be enhanced and rise above the resting pressure, accompanied by bursts of EMG activity.

In patients with a colostomy, smaller increments of air are required to elicit this rectosphincteric reflex, the pressure within the rectum often remaining high.

\section{HIRSCHSPRUNG'S DISEASE}

In the resting trace, the rectum often shows spontaneous waves of varying amplitude and frequency (Fig. 4). The internal sphincter zone pressure is a little higher, between 32 and $64 \mathrm{~cm}$ water, with a mean of $44 \mathrm{~cm}$ water. The rhythmical activity is more pronounced with an amplitude of 15 to $20 \mathrm{~cm}$ and tends to be a little slower at 8 to 12 /minute.

On rectal distension, a comparable increment of air will produce a high rectal pressure which slowly falls as the bowel wall adapts, superimposed on which are slow repeated waves. The internal sphincter shows a total absence of inhibition, with the pressure never falling below resting levels, and on occasion rising slightly with an enhanced rhythmical activity.

Deflation produces a return to resting conditions. The external sphincter appears to behave normally throughout. The presence of a colostomy increases further the high rectal pressure seen after adaptation to the inflated balloon.

\section{Ultrashort segment Hirschsprung's disease}

Where the rectal balloon lies above the aganglionic zone in normally innervated bowel, a normal rectal

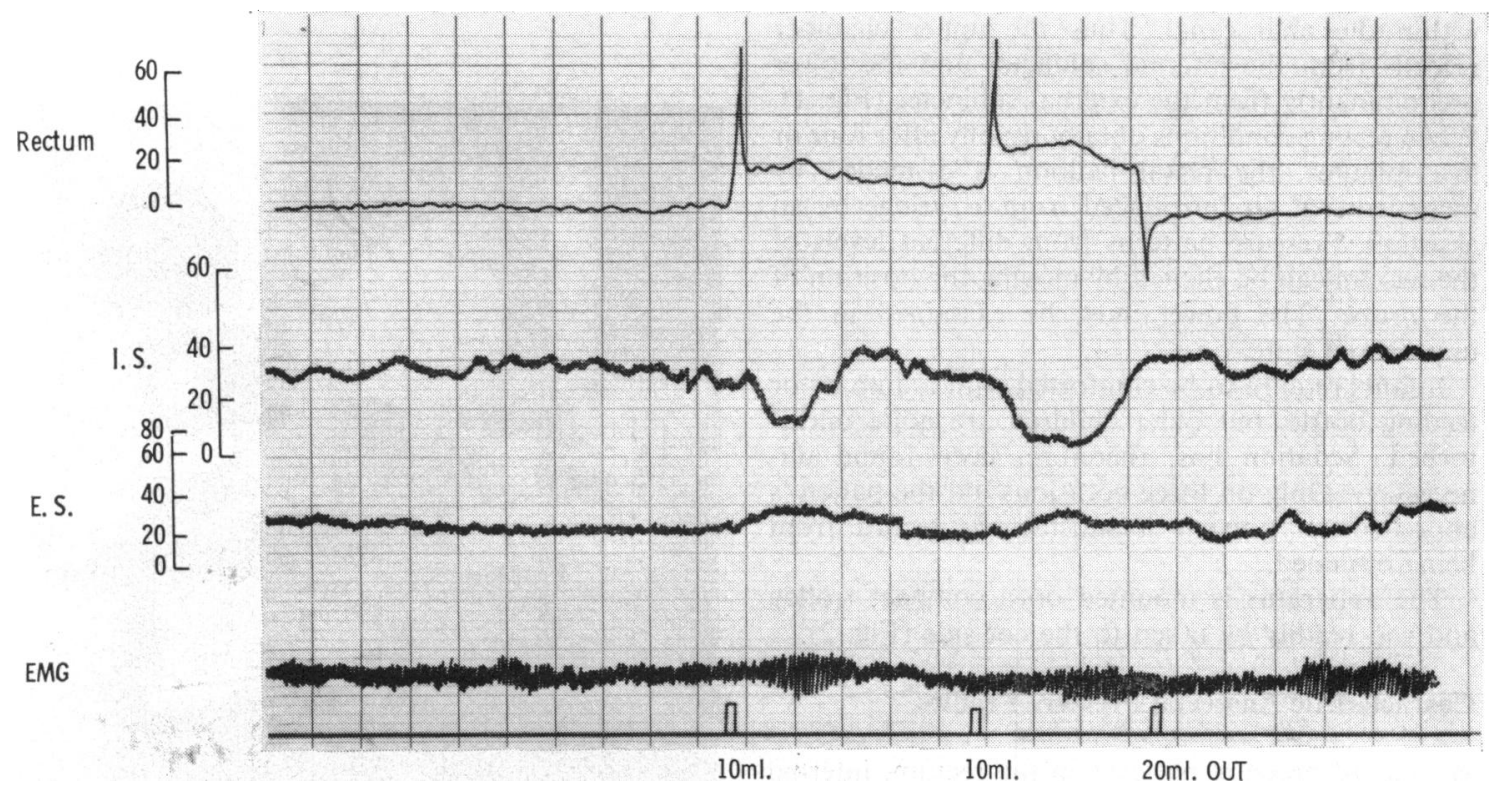

Fig. 3 Normal trace. An asymptomatic patient aged 8 months. The internal sphincter (I.S.) is inhibited as the rectum is stimulated. Deflation produces a brisk return of rhythmical activity. The external sphincter zone (E.S.) reflects the EMG changes. Pressures are in $\mathrm{cm} \mathrm{H}_{2} \mathrm{O}$. Vertical lines represent six-second intervals. 
Rectum ${ }_{20}^{40}{ }_{20}^{80} 5$

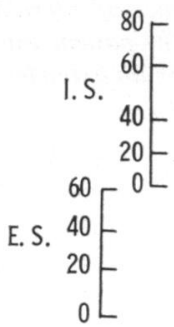

EMG

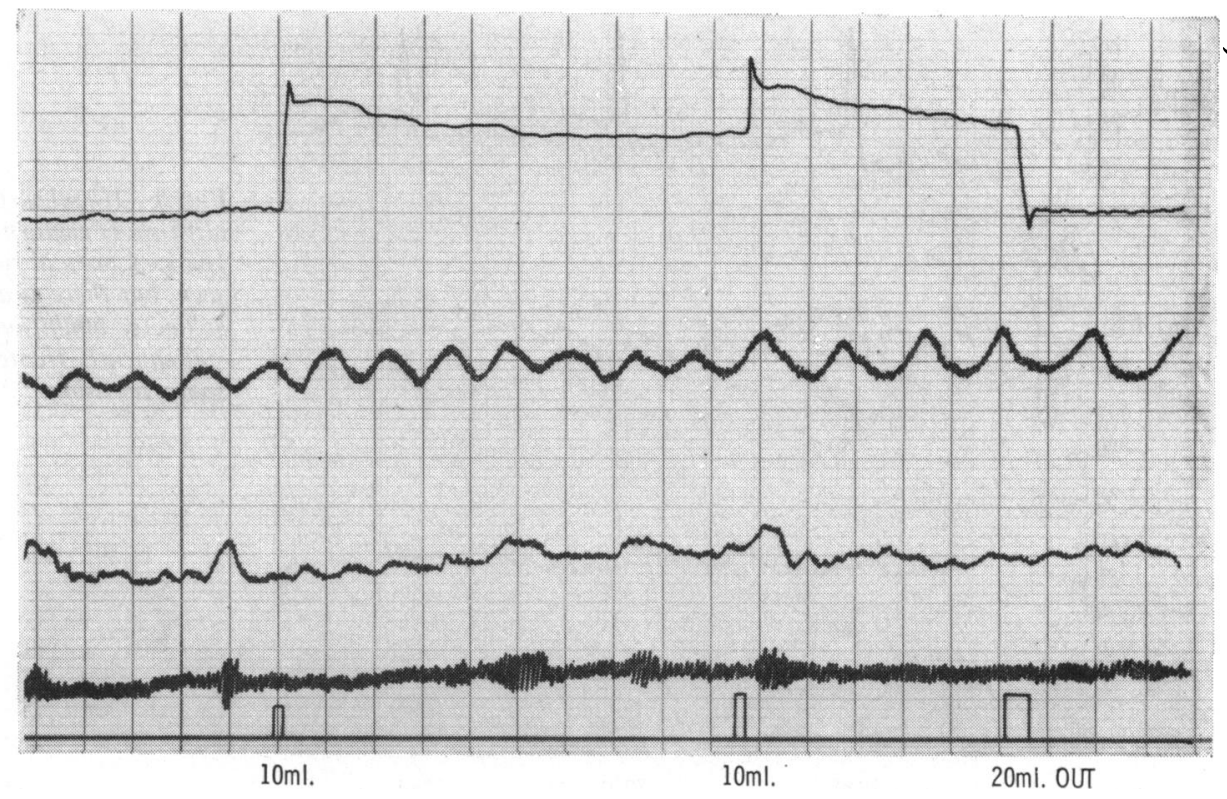

20mi. OUT

Fig. 4 Hirschsprung's disease. Patient aged 5 months. Similar increments of air produce a high rectal pressure with a characteristic slow fall and repeated rectal waves. The internal sphincter shows very pronounced rhythmical activity which is here enhanced by rectal stimulation.

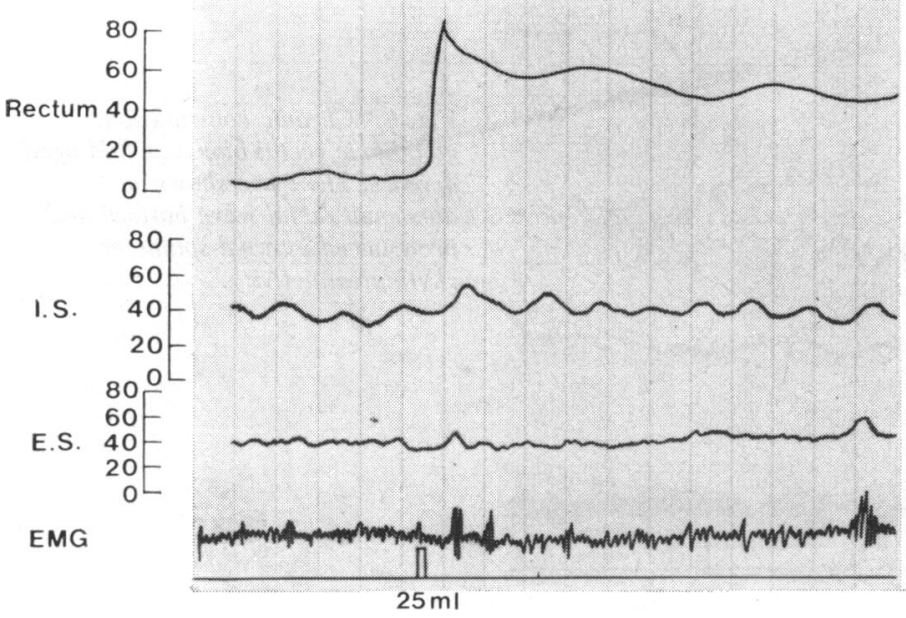

Fig. 4a Hirschsprung's disease. Patient aged 3 years. The resting trace may show well defined spontaneous rectal waves which are enhanced by rectal balloon inflation.

pattern in conjunction with typical Hirschsprung characteristics of the internal sphincter may be seen (Fig. 5).

\section{OTHER PATIENTS}

The patients we have examined have presented with a variety of bowel disorders, and we are now recognizing several trace patterns which, although not characteristic of Hirschsprung's disease, cannot be described as normal. Figure 6 illustrates a child with chronic constipation, in whom the internal sphincter activity is very pronounced, and only slightly suppressed by rectal distension on one occasion. Figure 7 shows the record from an infant aged 3 days with functional intestinal obstruction following respiratory distress at birth. Anal canal pressures are 


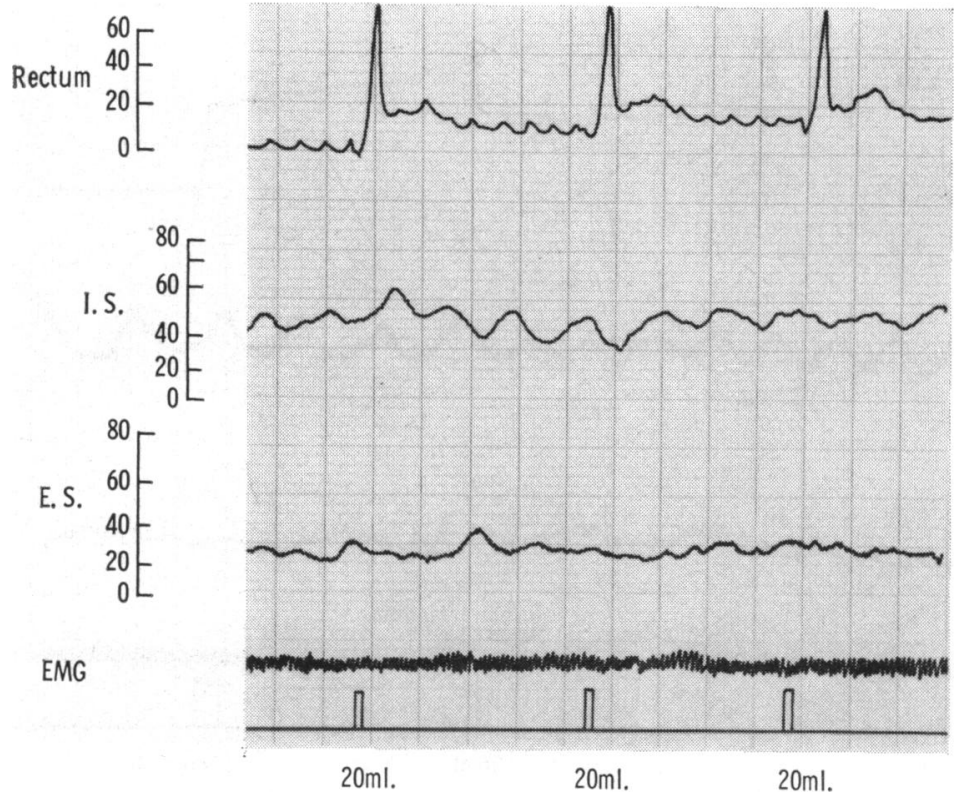

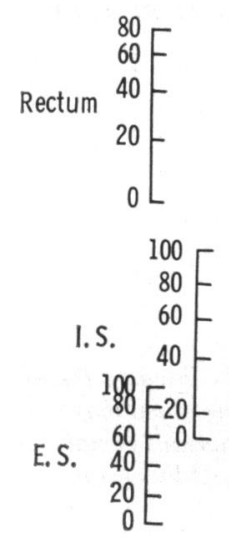

EMG
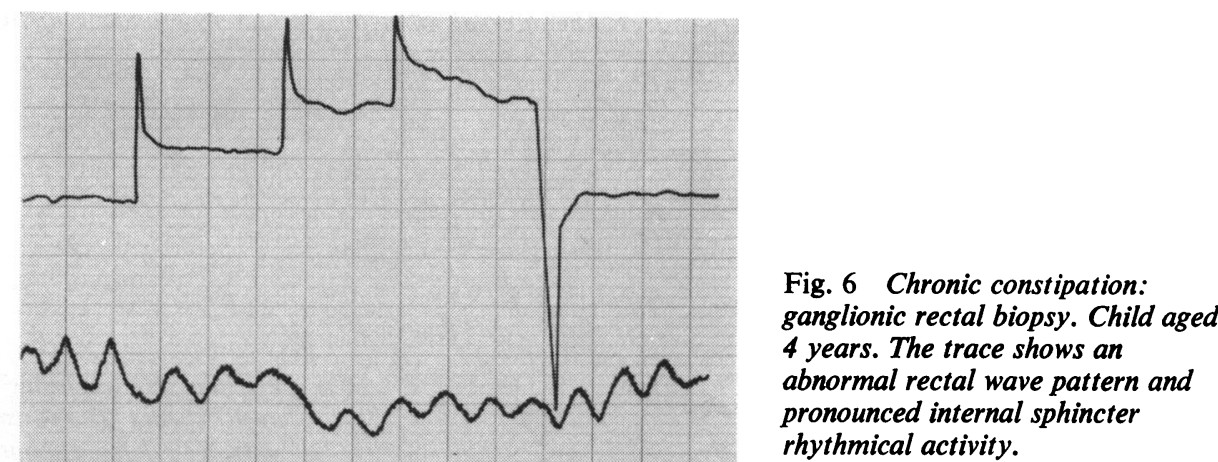

Fig. 6 Chronic constipation: ganglionic rectal biopsy. Child aged 4 years. The trace shows an pronounced internal sphincter rhythmical activity.
Fig. 5 Hirschsprung's disease: ultra-short segment. Child aged 4 years. A normal rectal pattern is seen, but there is absence of internal sphincter inhibition. The patient was subsequently treated by an extended sphincterotomy. abnormally low with a total absence of both rhythmical activity and the rectrosphincteric reflex. As the signs of obstruction subsided, the trace reverted to a normal pattern.

\section{Interpretation of the Pressure Profiles}

When a trace characteristic of the normal or
Hirschsprung pattern is obtained, the diagnosis or exclusion of Hirschsprung's disease may be confidently made. In practice, however, patients were examined because of some clinical bowel abnormality, and consequently a typically 'normal' trace was rarely seen.

We have found diagnosis based on an 'all or none' rectosphincteric reflex alone to be unreliable, only 

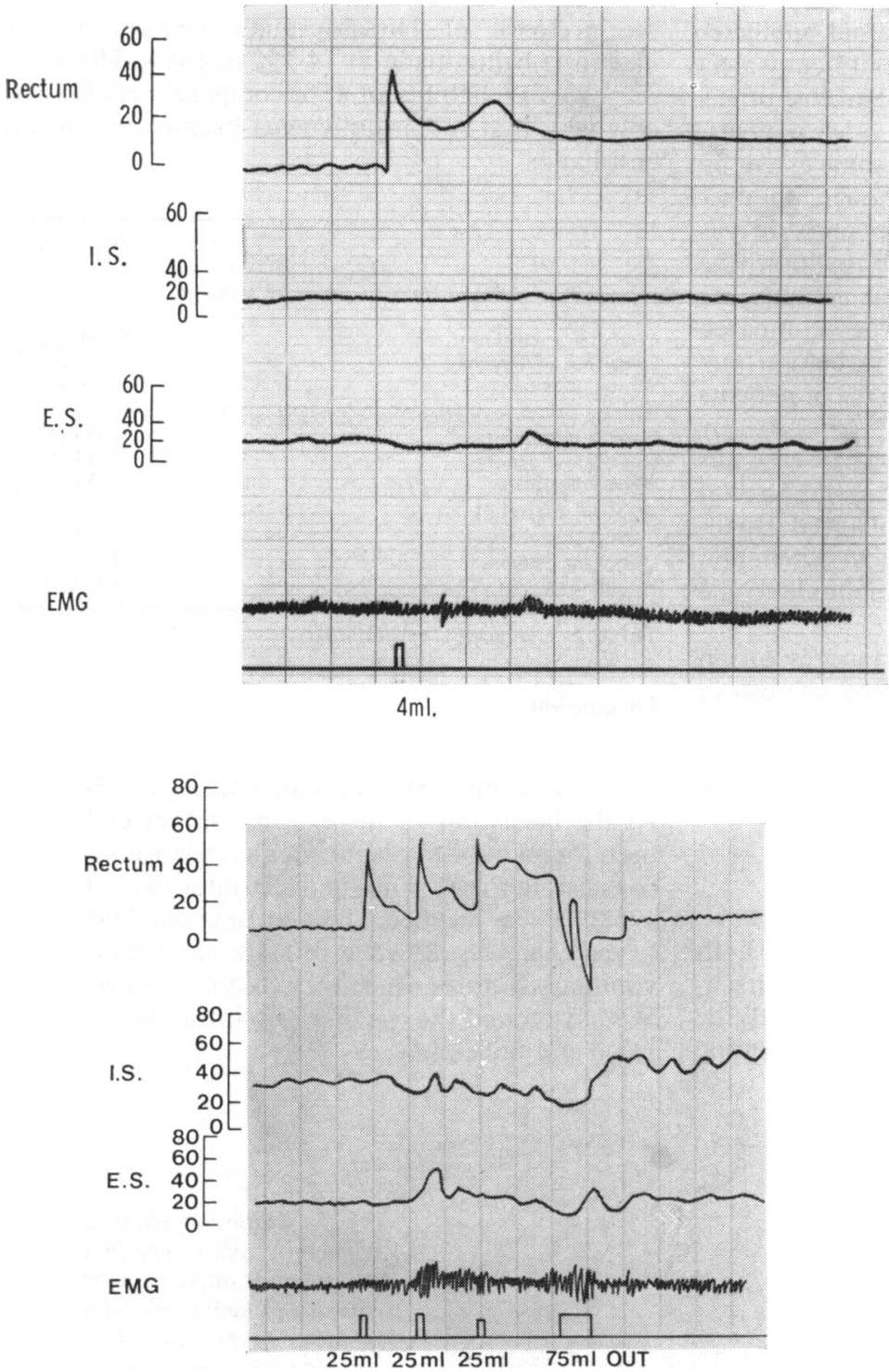

Fig. 7 Functional intestinal obstruction. Patient aged 3 days. Respiratory distress at birth. The anal canal pressure is low, and the internal sphincter rhythmical activity is suppressed. The rectosphincteric reflex is absent.

Fig. 8 Failure to elicit the rectosphincteric reflex. The lower chamber pressure (E.S.) and EMG indicate voluntary activity in a restless patient, which elevates and masks the internal sphincter trace.
$67 \%$ of our non-Hirschsprung cases showing a clear fall in internal sphincter pressure of more than $10 \mathrm{~cm}$ water. Failure to elicit the reflex in the remainder was due to an inadequate stimulus to the rectum, the presence of faeces alongside the probe masking pressure changes, overstimulation producing a voluntary external sphincter contraction, or, most commonly, failure to wait for resting conditions to obtain.

Conversely, in $26 \%$ of patients with proven Hirschsprung's disease, an apparently positive rectosphincteric reflex was obtained, the fall in anal canal pressure probably being due to movement of the probe, relaxation of external sphincter tone, or the passage of faeces.

In order to improve our reliability, we pay regard to the overall trace pattern throughout the examination. Spontaneously occurring rectal waves were seen in the resting trace in $52 \%$ of patients with Hirschsprung's disease. These were usually rendered more obvious by inflating the rectal balloon (Fig. 4a). In only $13 \%$ of non-Hirschsprung patients were spontaneous rectal waves seen in the resting trace, inflation of the balloon usually being followed by a 
single rectal wave. Pronounced internal sphincter rhythmical activity (an amplitude over $15 \mathrm{~cm}$ water) was seen in $94 \%$ of patients with Hirschsprung's disease. This was also seen in $17 \%$ of other patients, but was then inevitably suppressed to some extent by adequate rectal stimulation, even though an unequivocal fall in mean internal sphincter pressure was not always seen. On deflating the balloon the resumed internal sphincter rhythmical activity was often initially of greater amplitude when compared with the resting trace immediately before any stimulation. This was clearly seen in $65 \%$ of patients without Hirschsprung's disease (Fig. 10). By contrast, in patients with Hirschsprung's disease this was never seen, and in those patients where rectal stimulation was accompanied by enhanced rhythmical activity of the internal sphincter, deflation resulted in a gradual return of the amplitude to the resting level.

We have, therefore, based our diagnoses on an overall assessment of rectal wave pattern, changes in internal sphincter pressure and rhythmical activity on inflation and deflation of the rectal balloon, rather than on a simple, all-or-none presence of the rectosphincteric reflex.

\section{Results}

Our accuracy in diagnosis is shown in Table I. In $90.8 \%$ of our patients who did not have Hirschsprung's disease the condition could confidently be excluded. We have been less confident in confirming the presence of Hirschsprung's disease, a tirm diagnosis being made in $74.3 \%$ of cases. However, our overall reliability of $85 \%$ compares very favourably with that of barium enema examination in the best hands.

No. of Cases

\begin{tabular}{lc}
\hline Patients Proven Not to Have Hirschsprung's Disease & \\
(65 Cases) & $59(90.8 \%)$ \\
Confident exclusion & $6(9 \cdot 2 \%)$ \\
Uncertain of diagnosis & $0(0 \%)$ \\
Wrong diagnosis & \\
Patients with Proven Hirschsprung's Disease (35 Cases) & \\
Confident diagnosis & $26(74.3 \%)$ \\
Uncertain diagnosis & $5(14.3 \%)$ \\
Wrong diagnosis & $4(11.4 \%)$ \\
All Patients (100 Cases) & \\
Correct diagnosis & $85(85 \%)$ \\
Uncertain diagnosis & $11(11 \%)$ \\
Wrong diagnosis & $4(4 \%)$ \\
\hline
\end{tabular}

Table I Accuracy of diagnosis

\section{Discussion}

We have simplified our apparatus and technique to as little as is compatible with a high degree of reliability. As the behaviour of the external sphincter has not been found to be of diagnostic significance we do not consider the use of needle electrodes (Tobon et al, 1968) to be justified. The surface electrodes and lower chamber record give sufficient indication of voluntary features which may confuse interpretation. We have found the use of a respiratory line cumbersome and unhelpful.
Rectum $\begin{aligned} & 60 \\ & 40 \\ & 20 \\ & 0\end{aligned}$

EMG

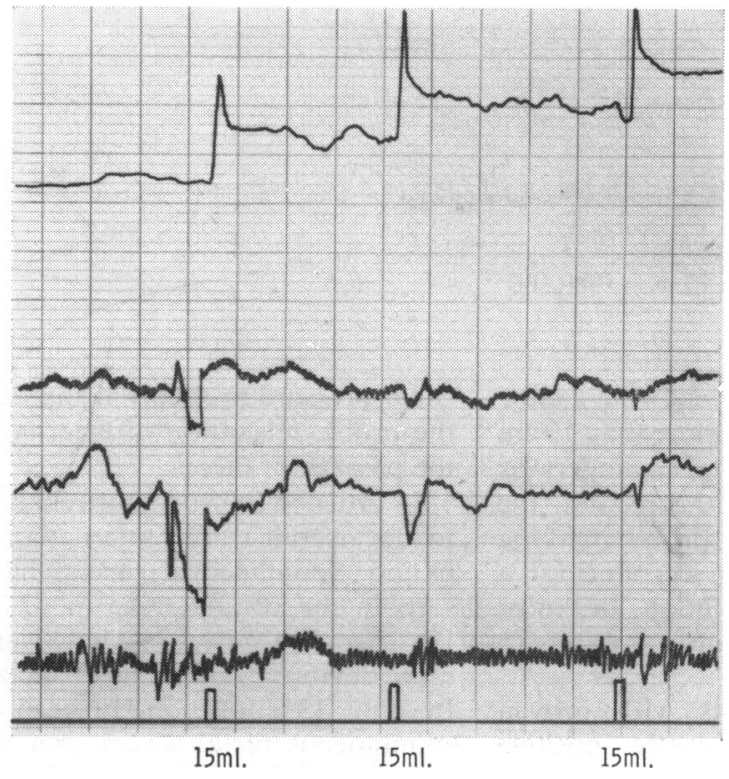

Fig. 9 Failure to elicit the rectosphincteric reflex. The first $20 \mathrm{ml}$ increment of air is an insufficient stimulus to inhibit the internal sphincter in the 9-month old patient with acquired megarectum. 


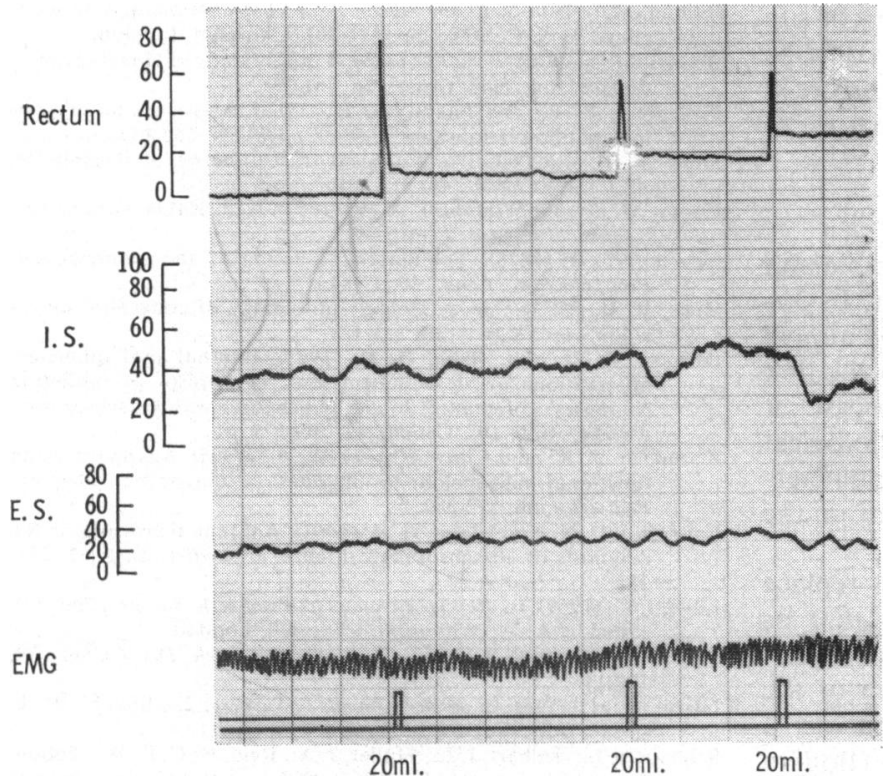

Fig. 10 Failure to elicit the rectosphincteric reflex. Voluntary activity masks the reflex, but deflation is followed by an enhanced rhythmical activity in the internal sphincter compared with the resting trace.

Careful attention to technique should ensure even greater reliability. The commonest reason for failing to elicit the rectosphincteric reflex is inflating the balloon before the anal canal has settled to a resting state (Fig. 8). The volume of air required to elicit the reflex is of critical importance and varies widely from one patient to another and can only be found by trial and error. Figure 9 shows the trace of a patient aged 9 months with a chronically dilated rectum where the anticipated stimulus produced no response. Conversely, overdistension will produce discomfort and a voluntary response which will mask the internal sphincter change. Rectal washouts, barium enema, or vigorous rectal examination within the previous 72 hours disturbs the sensitivity of the anorectum, resting conditions never being obtained.

We are attracted by the simple, self-retaining device developed by Ustach, Tobon, and Schuster (1969). However, we consider the unobtrusiveness within the anal canal of our fine probe to be of great importance in obtaining satisfactory resting conditions. We have found an ability to move the probe to record rectal pressures at different levels has proved valuable on several occasions in giving an indication of the length of the zone of aganglionosis, by detecting the level at which the rectal pressure trace records the typical 'spike-and-wave' response to distension of normally innervated bowel. Furthermore, a continuous record of the internal sphincter rhythmical activity has enabled us to separate anorectal stenosis from Hirschsprung's disease, as well as to improve our diagnostic reliability.
The recognition of the absence of the rectosphincteric reflex in the first days of life in the stressed infant (Howard and Nixon, 1968) may make this test unreliable in the first week of life. However, both our premature infants and eight other patients examined between 3 and 7 days of age all showed the reflex to be present. Our clearly abnormal recordings from the internal sphincter in some cases of chronic constipation (Fig. 6) indicate our incomplete understanding of the pathology in these patients (Duhamel, 1969).

\section{Comparison with Other Diagnostic Methods}

Barium enema examination may be difficult to interpret, particularly in long segment disease where delayed films up to 48 hours may be required (Berdon and Baker, 1965), in very short segment cases, and in patients with a colostomy (Lillie, 1969). A confident radiological diagnosis was made in $83 \%$ of our 59 patients who had a barium enema. In five patients $(8.5 \%)$ there was some uncertainty. In a further five cases, one of total aganglionosis of the colon and four of very short segment disease, the radiological diagnosis was incorrect but correctly made by our pressure studies.

Rectal biopsy is not without its complications (Swenson et al, 1955) and mortality (Fraser and Wilkinson, 1967) as well as difficulties in interpretation (Nixon, 1966), which may lead to inappropriate treatment (Kottmeier and Clatworthy, 1965). It is salutary to record that in seven of 49 of our patients 
subjected to rectal biopsy, either full-thickness or sub mucosal, with paraffin section, no diagnosis could be reached on the first specimen, either through errors in technique in taking the biopsy or in interpretation.

\section{Conclusion}

We have found this method of diagnosis simple and quite safely employed in the very ill patient. In our hands it is as reliable as a barium enema, and almost as likely to provide the correct diagnosis as the first rectal biopsy. In ultrashort segment disease it appears to be more reliable than radiological methods, and in two cases an extended sphincterotomy was performed as definitive treatment on the basis of our test with satisfactory results.

We do not feel that our method can yet replace biopsy as a final arbiter of diagnosis, but that its place is alongside radiological and histological methods when there is a clinical suggestion of the diagnosis of Hirschsprung's disease, and as a screening test where there is a family history (Bodian and Carter, 1963) of Hirschsprung's disease.

\section{I.A. acknowledges a grant from the Medical Research Council.}

Correspondence should be addressed to Ian Aaronson, The Middlesex Hospital, London, W1.

References

Berdon, W. E., and Baker, D. H. (1965). The roentgenographic diagnosis of Hirschsprung's disease in infancy. Amer. $J$. Roentgenol., 93, 432-446.
Bodian, M. (1960). In Recent Advances in Clinical Pathology, Series 3, edited by S. C. Dyke, pp. 384-392. Churchill, London.

Bodian, M., and Carter, C. O. (1963). A family study of Hirschsprung's disease. Ann. hum. Genet., 26, 261-271.

Callaghan, R. P., and Nixon, H. H. (1964). Megarectum: physiological observations. Arch. Dis. Childh., 39, 153-157.

Duhamel, B. (1969). Physio-pathology of the internal anal sphincter. Arch. Dis. Childh., 44, 377-281.

Fraser, G. C., and Wilkinson, A. W. (1967). Neonatal Hirschsprung's disease. Brit. med. J., 3, 7-10.

Gowers, W. R. (1878). The automatic action of the sphincter ani. Proc. roy. Soc. Lond., 26, 77-84.

Hiatt, R. B. (1951). The pathologic physiology of congenital megacolon. Ann. Surg., 133, 313-320.

Howard, E. R., and Nixon, H. H. (1968). Internal anal sphincter: observations on development and mechanism of inhibiting responses in premature infants and children with Hirschsprung's disease. Arch. Dis. Childh., 43, 569-578.

Kottmeier, P. K., and Clatworthy, H. W., Jr. (1965). Aganglionic and functional megacolon in children-a diagnostic dilemma. Pediatrics, 36, 572-582.

Lawson, J. O. N., and Nixon, H. H. (1967). Anal canal pressures in the diagnosis of Hirschsprung's disease. J. paediat. Surg., 2, 544552.

Lillie, J. G. (1969). In Recent Advances in Paediatric Surgery, 2nd ed., edited by A. W. Wilkinson. Churchill, London.

Nixon, H. H. (1964). Hirschsprung's disease. Arch. Dis. Childh., 39, 109-115.

Nixon, H. H. (1966). In Modern Trends in Surgery, 2, edited by W. T. Irvine. Butterworths, London.

Schnaufer, L., Talbert, J. L., Haller, J. A., Reid, N. C. R. W., Tobon, F., and Schuster, M. M. (1967). Differential sphincteric studies in the diagnosis of ano rectal disorders of childhood. J. paediat. Surg., 2, 538-543.

Swenson, O., Rheinlander, H. F., and Diamond, I. (1949). Hirschsprung's disease: a new concept of the etiology. New Engl. J. Med., 241, 551-556.

Swenson, O., Fisher, J. H., and MacMahon, H. E. (1955). Rectal biopsy as an aid in the diagnosis of Hirschprung's disease. New Engl. J. Med., 253, 632-635.

Tobon, F., Reid, N. C. R. W., Talbert, J. L., and Schuster, M. M. (1968). Non-surgical test for the diagnosis of Hirschsprung's disease. New Engl. J. Med., 278, 188-194.

Ustach, T. J., Tobon, F., and Schuster, M. M. (1969). Simplified method for diagnosis of Hirschsprung's disease. Arch. Eis. Childh., 44, 694-697. 